\title{
Orally administered octacosanol improves some features of high fructose-induced metabolic syndrome in rats
}

\author{
Koji Ohashi, ${ }^{1}$ Yoshiji Ohta, ${ }^{2, *}$ Hiroaki Ishikawa, ${ }^{1}$ and Akira Kitagawa ${ }^{3}$ \\ ${ }^{1}$ Department of Biomedical and Clinical Sciences, Faculty of Clinical Technology and ${ }^{2}$ Department of Chemistry, Fujita Health University School of Medicine, \\ Fujita Health University School of Health Sciences, 1-98 Dengakugakubo, Kutsukake-cho, Toyoake, Aichi 470-1192, Japan \\ ${ }^{3}$ Department of Nutrition, Faculty of Health Wellness, Shigakkan University, 55 Nakoyama, Yokonemachi, Obu, Aichi 474-8651, Japan
}

(Received 31 March, 2020; Accepted 8 April, 2020; Published online 10 July, 2020)

\begin{abstract}
We examined whether orally administered octacosanol, a longchain aliphatic saturated alcohol, improves the features of high fructose-induced metabolic syndrome in rats. Five-week-old rats were fed a high fructose diet containing $60 \%$ fructose for 3 weeks. Then, the high fructose fed rats received a daily single oral administration of octacosanol (10 or $100 \mathrm{mg} / \mathrm{kg}$ body weight) with high fructose feeding for one week. Three- or four-week high fructose feeding increased insulin resistance, serum insulin, triglyceride, total cholesterol, free fatty acids, uric acid, and lipid peroxide concentrations, and hepatic triglyceride and cholesterol contents significantly and decreased serum high-density lipoprotein cholesterol and adiponectin concentrations significantly but did not affect blood pressure and hepatic lipid peroxide and reduced glutathione contents. Four-week high fructose feeding decreased hepatic ascorbic acid content significantly. Oral administration of octacosanol (10 or $50 \mathrm{mg} / \mathrm{kg}$ body weight) to high fructose-fed rats for the last 1-week fructose diet feeding attenuated these changes except serum insulin level and insulin resistance significantly and increased hepatic reduced glutathione content significantly. The higher dose of Oct decreased hepatic lipid peroxide content significantly. These results indicate that orally administered octacosanol improves dyslipidemia, hyperuricemia, hypoadiponectinemia, and oxidative stress associated with the features of high fructose-induced metabolic syndrome rats.
\end{abstract}

Key Words: octacosanol, high fructose diet feeding (rats), metabolic syndrome, dyslipidemia, oxidative stress

$\mathrm{O}$ ctacosanol (Oct) $\left[\mathrm{CH}_{3}\left(\mathrm{CH}_{2}\right)_{26} \mathrm{CH}_{2} \mathrm{OH}\right]$ is a major component of the natural mixture of long-chain primary aliphatic saturated alcohols, polycosanol, and it is commonly present in the natural wax extracted from various plant parts such as fruits, leaves, and barks. ${ }^{(1)}$ Kato et al. ${ }^{(2)}$ have demonstrated that dietary supplementation of Oct ( $10 \mathrm{~g} / \mathrm{kg}$ diet $)$ to rats fed a high fat diet for 40 days decreases serum triglyceride level, enhances serum free fatty acids (FFA) level, and prevents an increase in hepatic triacylglycerol level probably through inhibition of hepatic phosphatidate phosphohydrolase and that this dietary Oct supplementation activates lipoprotein lipase in the perirenal adipose tissue and the total oxidation rate of fatty acids in the muscle. Xu et $a l^{(3)}$ have demonstrated that dietary supplementation of Oct $(10 \mathrm{~g} / \mathrm{kg}$ diet $)$ to apolipoprotein E-knockout mice fed a Westerntype diet containing $0.2 \%$ cholesterol for 12 weeks causes a marked decrease in triglyceride level with a slight increase in total cholesterol level in the plasma. We have reported that dietary administration of Oct ( 10 or $50 \mathrm{mg} / \mathrm{kg}$ body weight per day) to type 2 diabetic KKAy mice for 5 weeks, starting at the time point of apparent diabetes occurrence, attenuates increased serum triglyceride and total cholesterol levels, hepatic triglyceride and cholesterol levels, and serum and hepatic lipid peroxide (LPO) levels and decreased hepatic reduced glutathione (GSH) level more efficiently at its higher dose than at its lower dose without affecting hyperglycemia, glucose intolerance, and hyperinsulinemia. ${ }^{(4)} \mathrm{A}$ recent report has shown that daily oral administration of Oct $(60 \mathrm{mg} / \mathrm{kg}$ body weight) to mice fed a high fat diet for 4 weeks prevents obesity and obesity-related metabolic disorders by activating thermogenic changes in the brown adipose tissue and that the 4-week oral administration of Oct attenuates obesity associated with the hepatic accumulation of triglyceride and cholesterol by improving hepatic lipid metabolism associated with lipogenesis and $\beta$-oxidation. ${ }^{(5)}$ In addition, we have reported that when rats treated once with carbon tetrachloride receive a single oral administration of Oct $(50 \mathrm{mg} / \mathrm{kg}$ body weight) after the occurrence of liver injury, a decrease in serum triglyceride level, and an increase in hepatic triglyceride level, not only liver injury progression but also further changes in serum and hepatic triglyceride levels are prevented and that a single oral administration of the same dose of Oct to normal rats reduces serum and liver triglyceride levels. ${ }^{(6)}$ Furthermore, we have reported that a single oral administration of Oct $(10,50$, or $100 \mathrm{mg} / \mathrm{kg}$ body weight) to rats treated once with carbon tetrachloride prevents the progression of acute liver injury by attenuating disrupted hepatic reactive oxygen species metabolism dose-dependently and that the single administration of Oct $(10,50$, or $100 \mathrm{mg} / \mathrm{kg}$ body weight) to normal rats causes a decrease in hepatic LPO level and an increase in hepatic GSH level in a dose-dependent manner. ${ }^{(7)}$

Metabolic syndrome (MetS), which is also known as syndrome $\mathrm{X}$ or insulin resistance syndrome, is characterized by the constellation of metabolic abnormalities including glucose intolerance, hyperinsulinemia, insulin resistance, central obesity, dyslipidemia, hyperuricemia, systemic and adipose tissue inflammation, and hypertension, which increases the risk for developing type 2 diabetes and cardiovascular disease..$^{(8,9)}$ Oxidative stress is also involved in the pathophysiology of MetS. ${ }^{(10,11)}$ It has been reported that the features of MetS such as hyperinsulinemia, insulin resistance, dyslipidemia, hypertension, and oxidative stress appear in humans with excessive fructose intake. ${ }^{(12-16)}$ It is known that rats with high fructose intake have the features of MetS, i.e., glucose intolerance, hyperinsulinemia, insulin resistance, dyslipidemia including hypertriglyceridemia and hypercholesterolemia, hyperuricemia, hypertension, and adipose tissue inflammation associated

To whom correspondence should be addressed.

E-mail: yohta@fujita-hu.ac.jp 
with adipokines such as adiponectin, leptin, and tumor necrosis factor- $\alpha .^{(17-22)}$ It is also known that oxidative stress is associated with the features of MetS in rats with high fructose intake. ${ }^{(17-19,21-25)}$ At present, however, there is no available information whether orally administered Oct improves the features of MetS such as hyperinsulinemia, insulin resistance, dyslipidemia, hyperuricemia, hypoadiponectinemia, hypertension, and oxidative stress in rats with high fructose intake.

In the present study, therefore, we examined whether daily oral Oct administration, starting after the appearance of the features of MetS, improves hyperinsulinemia, insulin resistance, dyslipidemia, hyperuricemia, hypoadiponectinemia, hypertension, and oxidative stress in rats fed a high fructose diet (HFD) in which $60 \%$ dextrose in regular rat chow was replaced by $60 \%$ fructose.

\section{Materials and Methods}

Materials. Oct (1-octacosanol), defatted bovine serum albumin, and bovine serum albumin were obtained from SigmaAldrich Japan Co. (Tokyo, Japan). L-Ascorbic acid, 5,5'-dithiobis(2-nitrobenzoic acid) (DTNB), 2,2'-dipyridyl, ethylenediaminetetraacetic acid (EDTA), GSH, 2-thiobarbituric acid (TBA), and other reagents of the highest grade were purchased from FUJIFILM Wako Pure Chemical Corporation (Osaka, Japan). All chemicals were used without further purification.

Animals. Male Wistar rats aged 4 weeks were purchased from Nippon SLC Inc. (Hamamatsu, Japan). The animals were maintained under daily controlled $12 \mathrm{~h}$-light and $12 \mathrm{~h}$-dark lighting cycle at $23^{\circ} \mathrm{C}$ and $50 \%$ humidity with free access to rat chow (Oriental MF, Oriental Yeast Co. Ltd., Tokyo, Japan) and water for one week prior to the experiment. All animals received humane care in compliance with the guidelines of the Management of Laboratory Animals in Fujita Health University, Japan. The animal experiment was approved by the Institutional Animal Care and Use Committee, and its approved protocol number was H0821.

HFD feeding and Oct administration. Rats aged 5 weeks were fed either a control diet (CD) or HFD for 3 and 4 weeks, because it is known that steady-state MetS in rats fed a diet rich in fructose is observed between 12- and 30-day diet feeding. ${ }^{(20)}$ The composition of CD was $60 \%$ dextrose, $20 \%$ milk casein, $5.2 \%$ soy oil, $3.5 \%$ mineral mixture, $1.0 \%$ vitamin mixture, $10.05 \%$ cellulose powder, $0.25 \%$ choline bicarbonate, and $0.00104 \%$ tertbutyl hydroquinone. The composition of HFD was the same except that $60 \%$ dextrose in CD was replaced by $60 \%$ fructose. Both CD and HFD were supplied by Oriental Yeast Co. Ltd. The pair feeding was performed to ensure the same daily calorie intake in rats fed CD and HFD. Namely, the diet intake of CD-fed rats was matched to that of HFD-fed rats during the feeding period. Oct was suspended in $1 \mathrm{ml}$ of $5 \%$ carboxymethyl cellulose. The Oct suspension was prepared daily just before its administration. The Oct suspension was orally administered to HFD-fed rats at a dose of $1 \mathrm{ml} / 100 \mathrm{~g}$ body weight between 10:00 and 11:00 once daily through a stomach tube for 1 week, starting at 3-week HFD feeding. The dose of Oct was 10 or $50 \mathrm{mg} / \mathrm{kg}$ body weight per day. This Oct administration used was determined according to our previous reports. ${ }^{(6,7)}$ Rats not administered with Oct received the same volume of vehicle once daily for the same period in the same manner. After fasting for $9 \mathrm{~h}$, both HFD-fed and CD-fed rats were sacrificed under anesthesia with pentobarbital at 3-week HFD feeding and HFD-fed rats with or without Oct administration and CD-fed rats were sacrificed under the same anesthetic condition at 4-week HFD feeding. Just before sacrifice, each rat was weighed and then blood was collected from each rat by cardiac puncture. Each collected blood was separated into serum by centrifugation. Immediately after sacrifice, each liver was perfused with ice-cold $0.9 \% \mathrm{NaCl}$ from the portal vein to remove residual blood in the tissue and then removed from the body. Each isolated liver was washed well in ice-cold $0.9 \% \mathrm{NaCl}$, blotted on a filter, and weighed. The liver weight was estimated as \% body weight, i.e., $\mathrm{g} / 100 \mathrm{~g}$ body weight. The serum and isolated liver of each rat were stored at $-80^{\circ} \mathrm{C}$ until use.

Biochemical determinations. Serum insulin and adiponectin were assayed using Rat insulin ELISA kit (Mereodia, Uppsala, Sweden) and Mouse/rat adiponectin ELISA kit (Otsuka Pharmaceutical Co., Tokyo, Japan), respectively. Serum glucose, triglyceride, total cholesterol, high-density lipoprotein (HDL)cholesterol, FFA, and uric acid were assayed using commercial kits, Glucose CII-Test Wako, Triglyceride R-Test Wako, Cholesterol E-Test Wako, HDL cholesterol Test Wako, NEFA CTest Wako, and Uric acid C-Test Wako (FUJIFILM Wako Pure Chemistry Corporation, Osaka, Japan), respectively. The determined serum glucose and insulin concentrations were used for the assessment of insulin resistance using homeostasis model assessment for insulin resistance (HOMA-IR) score. HOMA-IR was calculated with the formula [fasting serum insulin $(\mu \mathrm{U} / \mathrm{ml}) \times$ fasting serum glucose $(\mathrm{mM}) / 22.5]$, as described by Matthews et al. ${ }^{(26)}$ Serum LPO was measured by the fluorometric TBA method of $\mathrm{Yagi}^{(27)}$ using tetramethoxypropane as a standard. The concentration of serum LPO is expressed as the amount of malondialdehyde (MDA) equivalents. In order to assay liver triglyceride and cholesterol, each liver tissue was homogenized in nine volumes of ice-cold $0.15 \mathrm{M} \mathrm{KCl}$ and then lipid in the homogenate was extracted in a mixture of chloroform and methanol $(2: 1 \mathrm{v} / \mathrm{v})$ according to the Folch method. ${ }^{(28)}$ The extract was dried at $45^{\circ} \mathrm{C}$ under nitrogen gas stream and then the extracted lipid was dissolved in a $5 \%$ solution of defatted bovine serum albumin. Triglyceride and cholesterol in the solution were assayed using the same commercial kits as used for the assays of serum triglyceride and total cholesterol. For the assays of hepatic LPO, GSH, and ascorbic acid, each liver tissue was homogenized in nine volumes of ice-cold $0.05 \mathrm{M}$ Tris- $\mathrm{HCl}$ buffer ( $\mathrm{pH} 7.4$ ) containing $1 \mathrm{mM}$ EDTA. Liver LPO was assayed by the colorimetric TBA method of Ohkawa et al. ${ }^{(29)}$ except that $1 \mathrm{mM}$ EDTA was added to the reaction mixture, using tetramethoxypropane as a standard. The content of hepatic LPO is expressed as the amount of MDA equivalents. Liver GSH was assayed by the DTNB method of Sedlak and Lindsay $^{(30)}$ using authentic GSH as a standard. Liver vitamin C was assayed by the 2,2'-dipyridyl method of Zannoni et al. ${ }^{(31)}$ using authentic L-ascorbic acid (vitamin C) as a standard.

Blood pressure measurement. Systolic blood pressure (SBP) was measured by the tail-cuff method using a sphygmomanometer BP-98A (Softron Co., Tokyo, Japan) in conscious rats at 1 day before sacrifice at 3- and 4-HFD feeding. After 3-day training sessions, the SBP of each rat was measured three times in a quiet room without disturbance of the rat. The measured SBP is expressed as the average value.

Statistical analysis. All results obtained are expressed as the mean \pm SD. The statistical analyses of the results were performed using a commercial Excel statistical package. Each mean value was compared by two-way analysis of variance (ANOVA) and Bonferroni/Dunn for multiple comparisons. The significance level was set at $p<0.05$.

\section{Results}

Effect of Oct on body weight and liver weight in rats fed HFD. There was no significant difference in body weight between HFD-fed and CD-fed rats at 3- and 4-week HFD feeding (Fig. 1A). Daily oral administration of Oct ( $10 \mathrm{or} 50 \mathrm{mg} / \mathrm{kg}$ body weight) for 1 week, starting at 3-week HFD feeding, had no effect on body weight in rats fed HFD for 4 weeks (Fig. 1B). There was no difference in liver weight (\% body weight, i.e., g/100 g body weight), between rats fed HFD for 3-weeks and the corresponding CD-fed rats, but the liver weight was significantly larger in rats fed HFD for 4 weeks than in the corresponding CD-fed rats (Fig. 1B). 
HFD feeding period
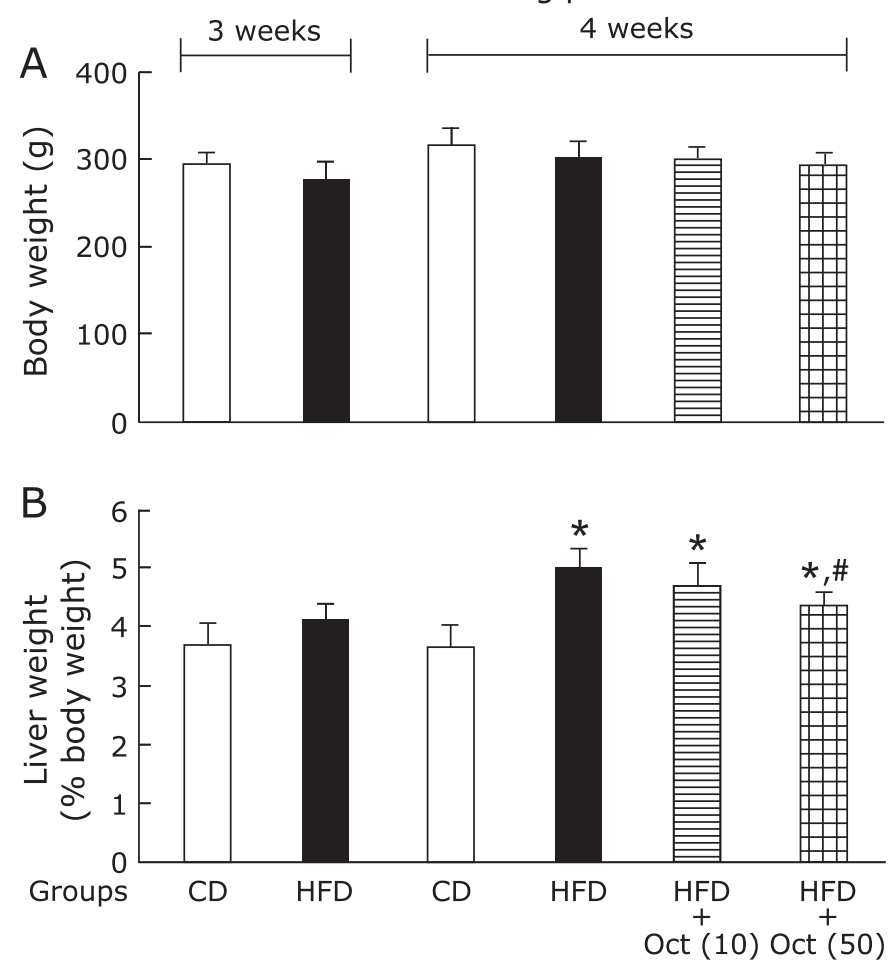

Fig. 1. Effect of orally administered Oct on body weight (A) and liver weight (B) in rats fed HFD. Rats were given CD or HFD in the same amount daily for 3 and 4 weeks. HFD was prepared by replacing $60 \%$ dextrose in regular rat chow with the same amount of fructose. Oct at a dose of 10 or $50 \mathrm{mg} / \mathrm{kg}$ body weight was orally administered every day for one week, starting at 3-week HFD feeding. Body weight was measured just before sacrifice and liver weight was measured after liver perfusion performed just after sacrifice. Liver weight is expressed as \% body weight (g/100 g body weight). CD, CD-fed rats; HFD, HFD-fed rats; HFD + Oct (10), HFD-fed rats with Oct administration $(10 \mathrm{mg} / \mathrm{kg}$ body weight); HFD + Oct (50), HFD-fed rats with Oct administration $(50 \mathrm{mg} / \mathrm{kg}$ body weight). Results are expressed as the mean $\pm S D(n=5$ for CD-fed rats; $n=8$ for rats fed HFD alone and HFD-fed rats with Oct administration). ${ }^{*} p<0.05$ vs the corresponding CD-fed rats; ${ }^{*} p<0.05$ vs HFD-fed rats without Oct administration.

This increase in liver weight (\% body weight) in rats fed HFD for 4 weeks was significantly reduced near to the level of the corresponding CD-fed rats by the administration of Oct at a dose of $50 \mathrm{mg} / \mathrm{kg}$ body weight, but not $10 \mathrm{mg} / \mathrm{kg}$ body weight, for the last one-week HFD feeding (Fig. 1B).

Effect of Oct on serum glucose and insulin concentrations and HOMA-IR score in rats fed HFD. There was no significant difference in serum glucose concentration between HFD-fed and CD-fed rats at 3- or 4-week HFD feeding (Fig. 2A). Serum insulin concentration and HOMA-IR score were significantly higher in HFD-fed rats than in CD-fed rats at 3- and 4-week HFD feeding, but the increases in serum insulin concentration and HOMA-IR score in rats fed HFD for 4 weeks were not affected by daily oral administration of Oct ( 10 or $50 \mathrm{mg} / \mathrm{kg}$ body weight) for the last one-week HFD feeding (Fig. 2B and C).

Effect of Oct on serum adiponectin and uric acid concentrations in rats fed HFD. Serum adiponectin concentration was significantly lower in HFD-fed rats than in CD-fed rats at 3- and 4-week HFD feeding, although the reduction of serum adiponectin concentration was significantly larger at 4-week HFD feeding than at 3 -week HFD feeding $(p<0.05)$ (Fig. 3A). Serum uric acid concentration was significantly higher in HFD-fed rats than in CD-fed rats at 3- and 4-week HFD feeding, although the increase in serum uric acid concentration was significantly larger at 4-week
HFD feeding period
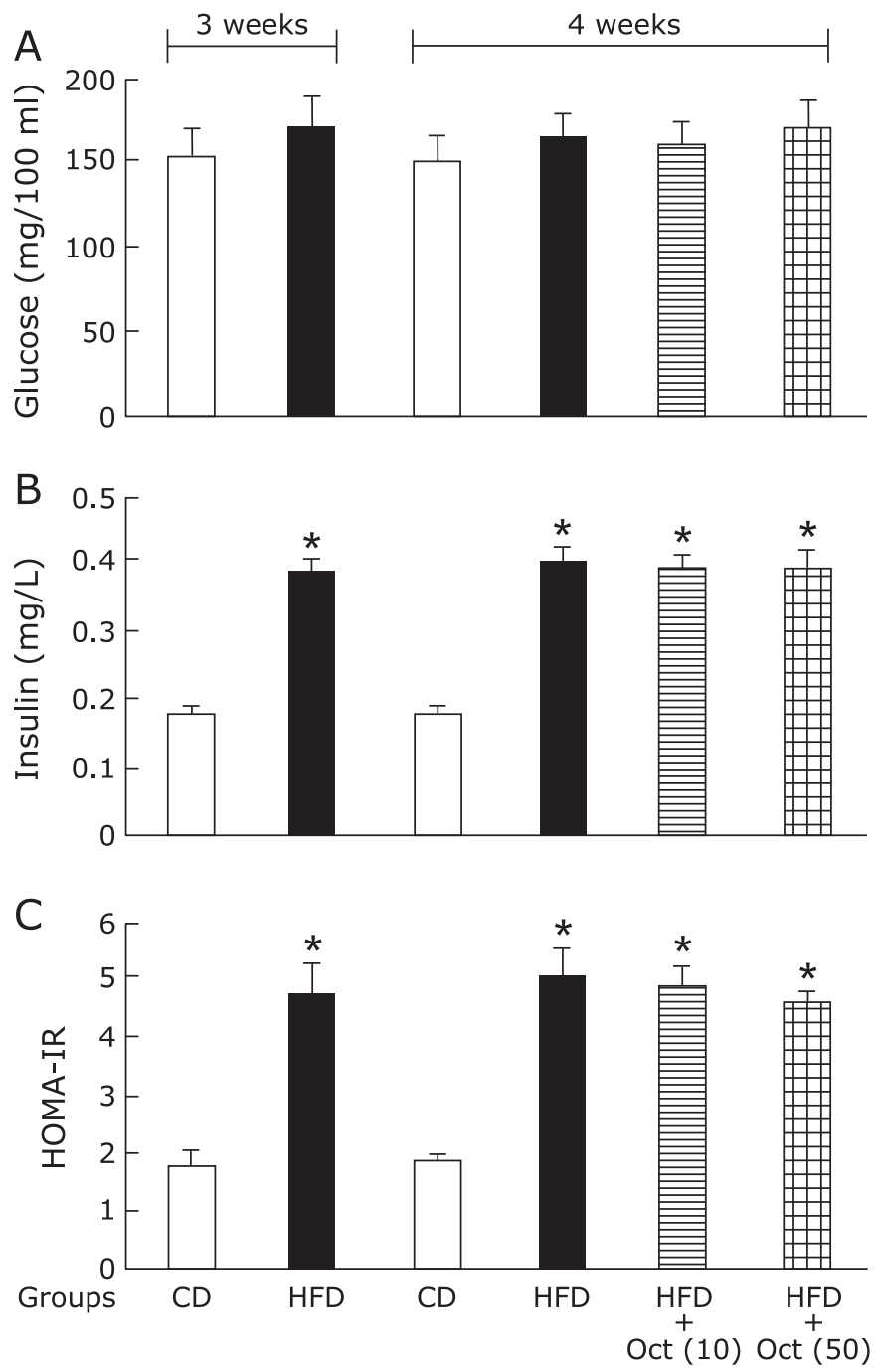

Fig. 2. Effect of orally administered Oct on serum glucose (A) and insulin (B) concentrations and HORMA-IR score (C) in rats fed HFD. Experimental condition and explanation are the same as described in the legend for Fig. 1 except that serum glucose and insulin concentrations and the score of HOMA-IR were determined as described in Materials and Methods. ${ }^{*} p<0.05$ vs the corresponding $C D$-fed rats.

HFD feeding than at 3 -week HFD feeding $(p<0.05)$ (Fig. 3B). Daily oral administration of Oct (10 or $50 \mathrm{mg} / \mathrm{kg}$ body weight) for the last one-week HFD feeding attenuated decreased serum adiponectin concentration and increased serum uric acid concentration at 4-week HFD feeding significantly, although these attenuating effects of Oct were significantly larger at its higher dose than at its lower dose $(p<0.05)$ (Fig. $3 \mathrm{~A}$ and $\mathrm{B}$ ).

Effect of Oct on serum FFA, triglyceride, total cholesterol, and HDL-cholesterol concentrations in rats fed HFD.

Serum FFA, triglyceride, and total cholesterol concentrations were significantly higher in HFD-fed rats than in CD-fed rats at 3- and 4-week HFD feeding (Fig. 4A-C). Moreover, the increases in serum FFA and total cholesterol concentrations tended to be higher at 4-week HFD feeding than at 3-week HFD feeding, while the serum triglyceride concentration was significantly higher at 4week HFD feeding than at 3-week HFD feeding $(p<0.05)$ and that concentration at 4-week HFD feeding was approximately 2-times higher than that at 3-week HFD feeding (Fig. 4A-C). The daily oral administration of Oct ( 10 or $50 \mathrm{mg} / \mathrm{kg}$ body weight) for the 

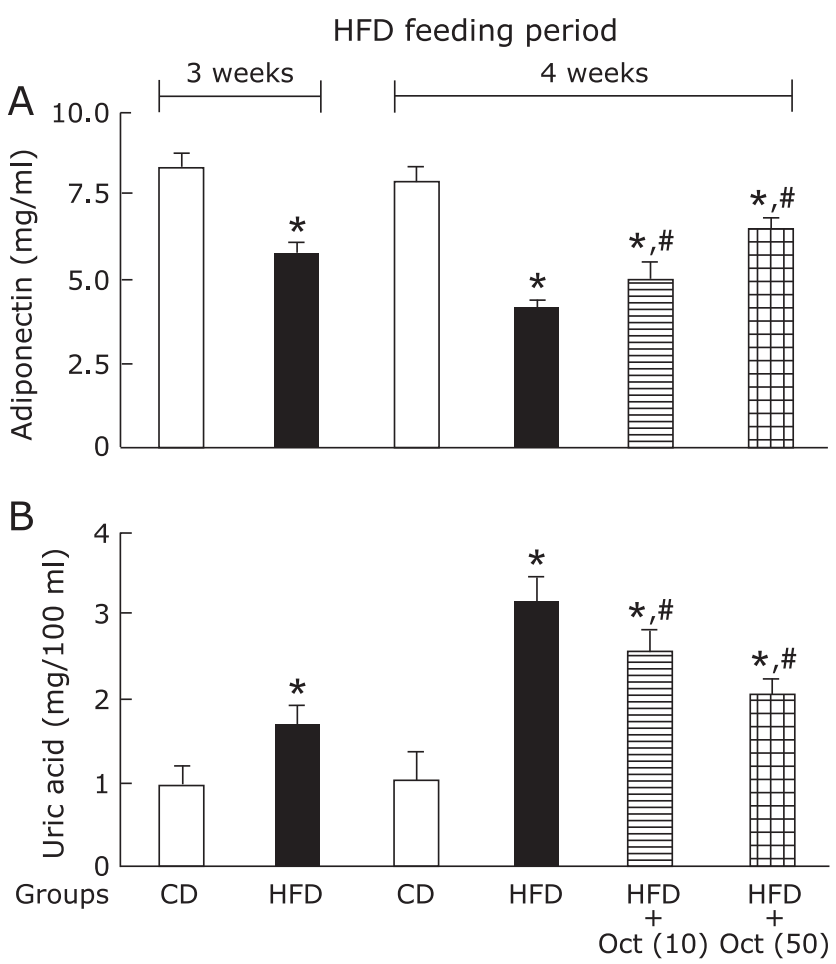

Fig. 3. Effect of orally administered Oct on serum adiponectin (A) and uric acid (B) concentrations in rats fed HFD. Experimental condition and explanation are the same as described in the legend for Fig. 1 except that serum adiponectin and uric acid concentrations were determined as described in Materials and Methods. ${ }^{*} p<0.05$ vs the corresponding $C D$-fed rats; ${ }^{*} p<0.05$ vs HFD-fed rats without Oct administration.
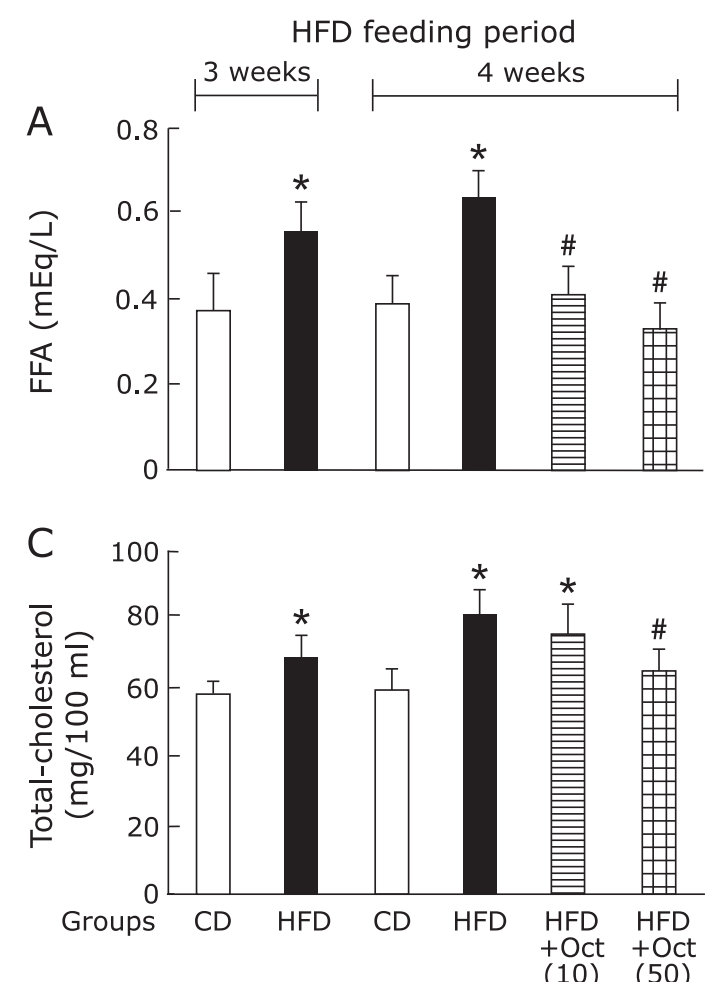

last one-week HFD feeding reduced the increases in serum FFA and triglyceride concentrations in rats fed HFD for 4 weeks significantly, although these reducing effects of Oct were more efficient at its higher dose than at its lower dose (Fig. 4A and B). As shown in Fig. 4C, the increased serum total cholesterol concentration in rats fed HFD for 4 weeks was significantly reduced near to that level of the corresponding CD-fed rats by the administration of Oct at a dose of $50 \mathrm{mg} / \mathrm{kg}$ body weight, but not $10 \mathrm{mg} / \mathrm{kg}$ body weight. Serum HDL-cholesterol concentration was significantly lower in HFD-fed rats than in CD-fed rats at 3- and 4-week HFD feeding, although that concentration tended to be lower at 4-week HFD feeding than at 3-week HFD feeding (Fig. 4D). The Oct administration at a dose of $50 \mathrm{mg} / \mathrm{kg}$ body weight, but not $10 \mathrm{mg} / \mathrm{kg}$ body weight, attenuated the decreased serum HDLcholesterol concentration at 4-week HFD feeding significantly (Fig. 4D).

Effect of Oct on hepatic triglyceride and cholesterol contents in rats fed HFD. Hepatic triglyceride and cholesterol contents were significantly higher in HFD-fed rats than in CD-fed rats at 3- and 4-week HFD feeding, although these increases tended to be larger at 4-week HFD feeding than at 3-week HFD feeding (Fig. 5). Daily oral administration of Oct $(10$ or $50 \mathrm{mg} / \mathrm{kg}$ body weight) for the last one-week HFD feeding attenuated the increased hepatic triglyceride content at 4-week HFD feeding significantly (Fig. 5A). In addition, the hepatic triglyceride content in HFD-fed rats with the higher dose of Oct was almost equal to that in the corresponding CD-fed rats, while the hepatic triglyceride content in HFD-fed rats with the higher dose of Oct tended to be less than that in the corresponding $\mathrm{CD}$-fed rats (Fig. 5A). The oral administration of Oct at a dose of 10 or $50 \mathrm{mg} / \mathrm{kg}$ body weight for the last one-week HFD feeding attenuated the increased hepatic cholesterol content at 4-week HFD feeding significantly and the attenuating effect of Oct tended to be larger at its higher dose than at its lower dose (Fig. 5B).
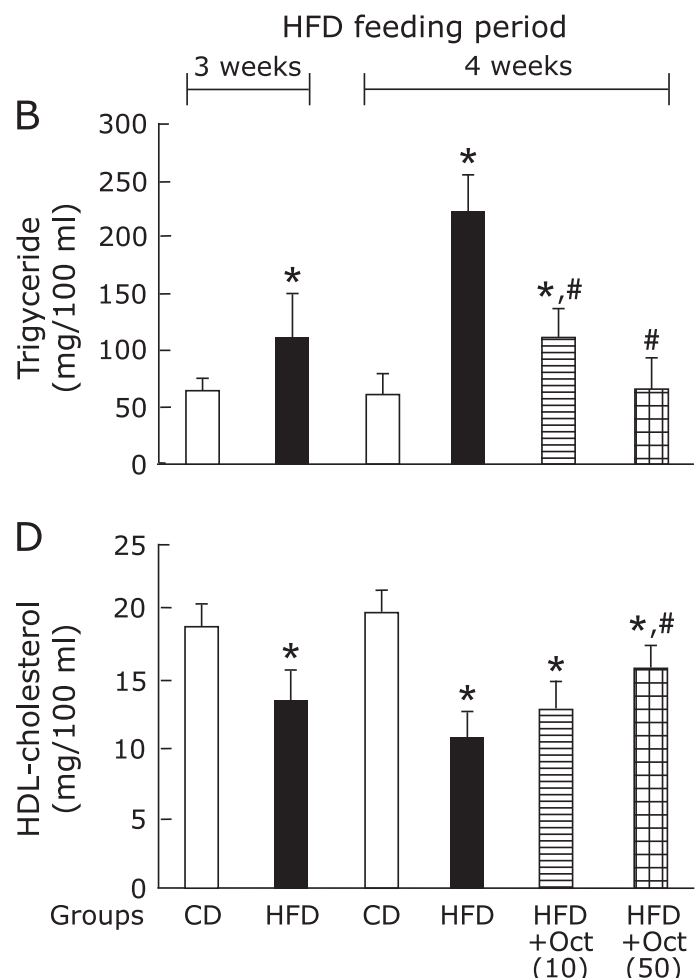

Fig. 4. Effect of orally administered Oct on serum FFA (A), triglyceride (B), total cholesterol (C), and HDL-cholesterol (D) concentrations in rats fed HFD. Experimental condition and explanation are the same as described in the legend for Fig. 1 except that serum FFA, triglyceride, total cholesterol, and HDL-cholesterol concentrations were determined as described in Materials and Methods. ${ }^{*} p<0.05$ vs the corresponding CD-fed rats; ${ }^{\#} p<0.05$ vs HFD-fed rats without Oct administration. 
HFD feeding period
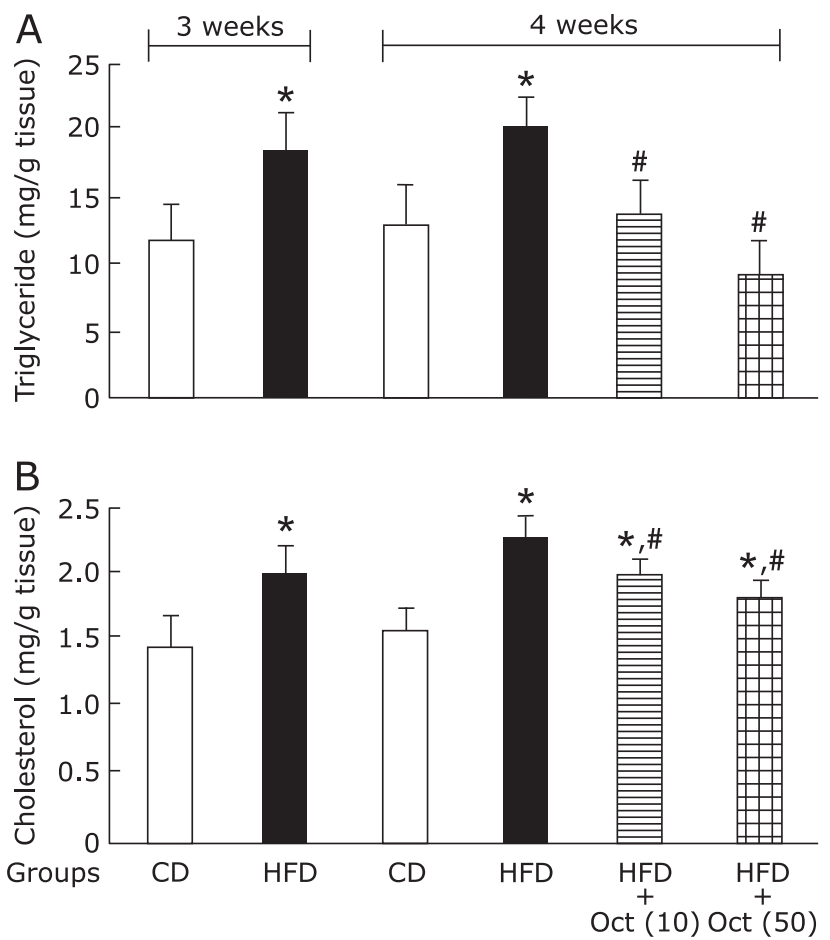

Fig. 5. Effect of orally administered Oct on hepatic triglyceride (A) and cholesterol (B) contents in rats fed HFD. Experimental condition and explanation are the same as described in the legend for Fig. 1 except that hepatic triglyceride and cholesterol contents were determined as described in Materials and Methods. ${ }^{*} p<0.05$ vs the corresponding CD-fed rats; ${ }^{*} p<0.05$ vs HFD-fed rats without Oct administration.
Effect of Oct on SBP in rats fed HFD. There was no significant difference in SBP between rats fed HFD for 3 and 4 weeks and the corresponding CD-fed rats (Fig. 6). Daily administration of Oct at a dose of 10 or $50 \mathrm{mg} / \mathrm{kg}$ body weight for the last oneweek HFD feeding had no effect on SBP at 4-week HFD feeding (Fig. 6).

Effect of Oct on serum LPO concentration and hepatic LPO, vitamin C, and GSH contents in rats fed HFD. Serum LPO concentration in rats fed HFD for 3 and 4 weeks was significantly higher than that in the corresponding CD-fed rats, although the increase in serum LPO concentration was significantly higher at 4 -week HFD feeding than at 3 -week HFD feeding $(p<0.05)$

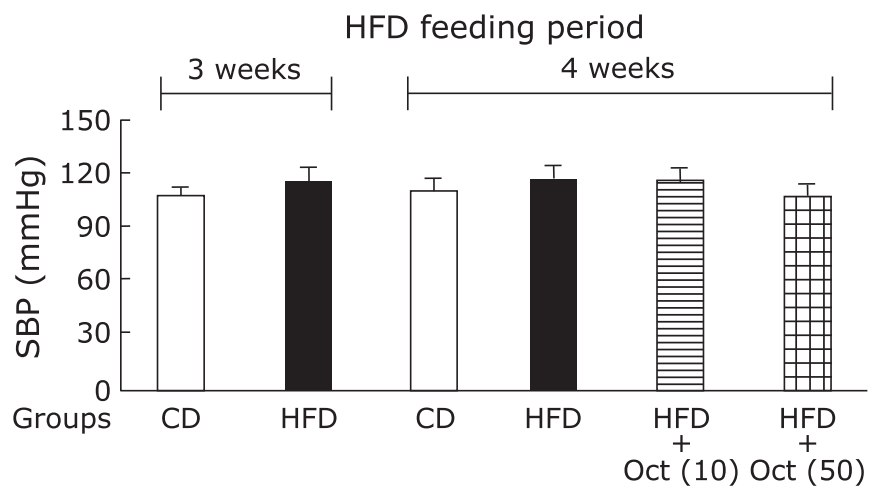

Fig. 6. Effect of orally administered Oct on SBP in rats fed HFD. Rats were given CD or HFD in the same amount daily for 3 or 4 weeks. Experimental condition and explanation are the same as described in the legend for Fig. 1 except that SBP was measured by the tail-cuff method using a sphygmomanometer in conscious rats at one day before sacrifice at 3- and 4-HFD feeding as described in Materials and Methods.
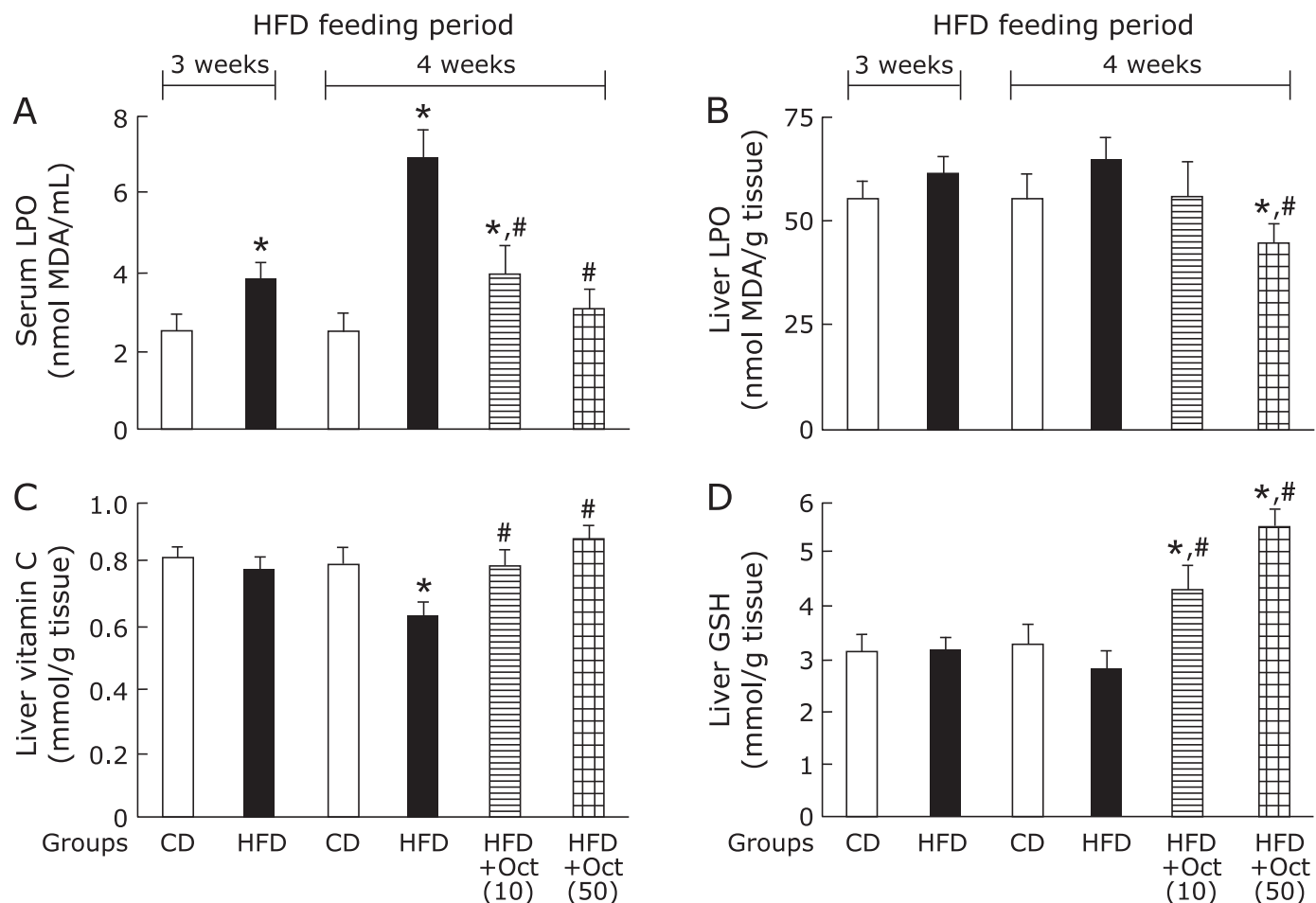

Fig. 7. Effect of orally administered Oct on serum LPO concentration (A) and liver LPO (B), vitamin C (C), and GSH (D) contents in rats fed HFD. Experimental condition and explanation are the same as described in the legend for Fig. 1 except that serum LPO concentration and hepatic LPO, vitamin C, and GSH contents were determined as described in Materials and Methods. ${ }^{*} p<0.05$ vs the corresponding CD-fed rats; ${ }^{*} p<0.05$ vs HFD-fed rats without Oct administration. 
(Fig. 7A). Daily oral Oct administration (10 or $50 \mathrm{mg} / \mathrm{kg}$ body weight) for the last one-week HFD feeding attenuated the increased serum LPO concentration at 4-week HFD feeding significantly, although the serum LPO concentration in HFD-fed rats with the higher dose of Oct was almost equal to that in the corresponding CD-fed rats (Fig. 7A). There was no significant difference in hepatic LPO content between rats fed HFD for 3 and 4 weeks and the corresponding $\mathrm{CD}$-fed rats, although the hepatic LPO content in rats fed HFD for 3 or 4 weeks tended to be higher than that in the corresponding CD-fed rats (Fig. 7B). The administration of Oct at a dose of $50 \mathrm{mg} / \mathrm{kg}$ body weight, but not $10 \mathrm{mg} / \mathrm{kg}$ body weight, to HFD-fed rats reduced the hepatic LPO content significantly (Fig. 7B). In addition, the hepatic LPO content in HFD-fed rats with the higher dose of Oct was significantly less than that in the corresponding CD-fed rats (Fig. 7B). Hepatic vitamin $\mathrm{C}$ content was significantly lower in HFD-fed rats than in the corresponding CD-fed rats at 4-week HFD feeding, but not at 3-week HFD feeding (Fig. 7C). The administration of Oct $(10$ or $50 \mathrm{mg} / \mathrm{kg}$ body weight) for the last 1 -week HFD feeding attenuated the decreased hepatic vitamin $\mathrm{C}$ content at 4week HFD feeding significantly (Fig. 7C). In addition, there was no significant difference in the hepatic vitamin $\mathrm{C}$ content between HFD-fed rats with the higher and lower dose of Oct and the corresponding CD-fed rats and the attenuating effect of Oct was significantly larger at its higher dose than at its lower dose $(p<0.05)$ (Fig. 7C). There was no significant difference in hepatic GSH content between HFD- and CD-fed rats at 3- and 4-week HFD feeding (Fig. 7D). The oral administration of Oct (10 or $50 \mathrm{mg} / \mathrm{kg}$ body weight) to HFD-fed rats caused a significant increase in the hepatic GSH content and the hepatic GSH content was significantly higher in HFD-fed rats with the higher and lower doses of Oct than in the corresponding CD-fed rats (Fig. 7D). In addition, the higher dose of Oct caused a significant higher increase in hepatic GSH content than the lower dose of Oct $(p<0.05)$ (Fig. 7D).

\section{Discussion}

It has been reported that feeding of a diet containing 60 or $65 \%$ fructose to rats for 3 or 4 weeks causes an increase in liver weight (\% body weight) without affecting body weight, ${ }^{(22,23,25,32)}$ although there is a report showing that feeding of a diet containing $65 \%$ fructose to rats for 3 weeks causes increases in both body weight and liver weight ( $\%$ body weight) ${ }^{(33)}$ In the present study, there was no significant difference in body weight between rats fed HFD containing $60 \%$ fructose for 3 or 4 weeks and the corresponding CD-fed rats, while liver weight (\% body weight) was significantly higher in rats fed HFD for 4 weeks than in the corresponding CD-fed rats. When Oct $(10$ or $50 \mathrm{mg} / \mathrm{kg}$ body weight) was orally administered to HFD-fed rats every day for 1 week, starting at 3-week HFD feeding, its higher dose attenuated the increase in liver weight ( $\%$ body weight) at 4-week HFD feeding significantly without affecting body weight. Thus, daily oral Oct administration was found to improve high fructoseinduced hepatic hypertrophy in rats.

It has been reported that healthy adult men with 2-week excessive fructose intake have the features of MetS such as hyperinsulinemia and insulin resistance. ${ }^{(34)}$ It is known that feeding of a diet containing 60 or $65 \%$ fructose to rats for 3 or 4 weeks causes hyperinsulinemia and/or insulin resistance as the features of MetS. ${ }^{(18,21-24)}$ In the present study, rats fed HFD for 3 and 4 weeks had significantly higher levels of serum insulin and HOMA-IR score, an index of insulin resistance, ${ }^{(26)}$ than the corresponding CD-fed rats, daily oral Oct administration $(10$ or $50 \mathrm{mg} / \mathrm{kg}$ body weight) for the last one-week HFD feeding did not affect the increases in serum insulin level and HOMA-IR score at 4-week HFD feeding. As to glycemic status in rats fed a diet rich in fructose for 3 and 4 weeks, there are several reports showing no change in glycemic status in rats fed a diet containing 58,60 or $65 \%$ fructose for 3 and/or 4 weeks, ${ }^{(22-24,32)}$ while there is a report showing hyperglycemia in rats fed a diet containing $60 \%$ fructose for 4 weeks. ${ }^{(21)}$ In the present study, serum glucose level in rats fed HFD for 3 or 4 weeks was not significantly different from that in the corresponding CD-fed rats. Thus, rats fed HFD for 3 and 4 weeks showed no hyperglycemia. Glycemic status in rats fed HFD for 4 weeks was not changed by daily oral administration of Oct $(10$ or $50 \mathrm{mg} / \mathrm{kg}$ body weight) for the last one-week HFD feeding. Thus, oral administration of Oct to HFD-fed rats at a dose of 10 or $50 \mathrm{mg} / \mathrm{kg}$ body weight was found to be unable to improve hyperinsulinemia and insulin resistance and to have no effect on glycemic status, as shown in type 2 diabetic KKAy mice given dietary Oct ( 10 or $50 \mathrm{mg} / \mathrm{kg}$ body weight) every day for 5 weeks. ${ }^{(4)}$

Our previous report ${ }^{(22)}$ has shown that hypoadiponectinemia and hyperuricemia occur as the features of MetS in rats fed a diet containing $60 \%$ fructose for 4 weeks. In the present study, rats fed HFD for 3 weeks showed a significant decrease in serum adiponectin concentration and a significant increase in serum uric acid concentration and these changes were further enhanced in rats fed HFD for 4 weeks. Thus, rats fed HFD for 3 and 4 weeks showed hypoadiponectinemia and hyperuricemia as the features of MetS. Daily oral administration of Oct $(10$ or $50 \mathrm{mg} / \mathrm{kg}$ body weight) to HFD-fed rats for the last one-week HFD feeding attenuated the decreased serum adiponectin concentration and increased serum uric acid concentration at 4-week HFD feeding significantly. These attenuating effects of Oct were larger at its higher dose than at its lower dose. Thus, orally administered Oct was found to improve hypoadiponectinemia and hyperuricemia associated with the features of MetS in HFD-fed rats. Baldwin et al. ${ }^{(36)}$ have demonstrated that excessive uric acid causes decreases in the expression levels of adiponectin mRNA and protein in 3T3-L1 adipocytes and the release of adiponectin from the adipocytes. Furthermore, the same authors have shown that decreased adiponectin production in the adipose tissue of obese Pound mice with MetS is attenuated when increased blood uric acid level is lowered by treatment with allopurinol, a xanthine oxidase inhibitor. ${ }^{(36)}$ Therefore, it is conceivable that the improvement of hypoadiponectinemia in HFD-fed rats by orally administered Oct is, at least in part, due to the improvement of hyperuricemia. However, the mechanisms by which orally administered Oct improves hyperuricemia and hypoadiponectinemia in rats fed HFD remains unclear at present. Therefore, these mechanisms are needed to be clarified in the future study.

Dyslipidemia including hypertriglyceridemia occurs in humans with high fructose intake. ${ }^{(12,13)}$ Indeed, it is known that feeding of a diet rich in fructose $(1.5 \mathrm{~g} / \mathrm{kg}$ body weight) over 4 weeks causes increases in plasma triacylglycerol and very low-density lipoprotein (VLDL)-triacylglycerol concentrations without causing ectopic lipid deposition and insulin resistance in healthy male adults. ${ }^{(37)}$ It has been demonstrated feeding of a diet containing 60 or $65 \%$ fructose to rats for 3 or 4 weeks causes increases in triglyceride and/or total cholesterol concentrations in the serum or plasma. ${ }^{(18,25,32,33)}$ We have reported that in addition to increases in serum triglyceride and total cholesterol concentrations, an increase in serum FFA concentration and a decrease in serum HDL-cholesterol concentration occur as the features of MetS in rats fed a diet $60 \%$ fructose for 4 weeks. ${ }^{(22)}$ In the present study, rats fed HFD for 3 and 4 weeks had significantly higher serum triglyceride, total cholesterol, and FFA concentrations than the corresponding CD-fed rats. The increase in serum triglyceride concentration was significantly higher at 4-week HFD than at 3week HFD feeding, while the increases in serum total cholesterol and FFA concentrations tended to be higher at 4-week HFD feeding than at 3-week HFD feeding. In addition, rats fed HFD for 3 and 4 weeks had a significantly lower serum HDL-cholesterol concentration than the corresponding $\mathrm{CD}$-fed rats and the decreased serum HDL-cholesterol concentration at 4-week HFD 
feeding tended to be lower than that at 3-week HFD feeding. Thus, rats fed HFD for 3 and 4 weeks showed dyslipidemia as the feature of MetS. Daily oral administration of Oct $(10$ or $50 \mathrm{mg} / \mathrm{kg}$ body weight) for the last one-week HFD feeding attenuated increased serum triglyceride and FFA concentrations at 4-week HFD feeding significantly. These attenuating effects of Oct were larger at its higher dose than at its lower dose. The daily oral administration of Oct $(50 \mathrm{mg} / \mathrm{kg}$ body weight) for the last oneweek HFD feeding attenuated increased serum total cholesterol concentration and decreased serum HDL-cholesterol concentration at 4-week HFD feeding. In addition, the serum FFA, triglyceride, and total cholesterol concentrations in HFD-fed rats with the higher dose of Oct were almost equal to those in the corresponding CD-fed rats. Thus, orally administered Oct was found to improve dyslipidemia associated with the feature of MetS in HFD-fed rats. It has been demonstrated that dietary Oct administration to rats fed a high fat diet for 20 days reduces serum triglyceride concentration probably by inhibiting hepatic phosphatidate phosphorylase which metabolizes phosphatidate to 1,2diacylglyceride in the pre-final step of triglyceride synthesis. ${ }^{(2)}$ Therefore, it is conceivable that orally administered Oct improves hypertriglyceridemia by inhibiting phosphatidate phosphorylase in the liver of HFD-fed rats. Qiao et al $^{\left({ }^{38)}\right.}$ have reported that an increase in plasma adiponectin concentration reduces fasting plasma triglyceride and FFA concentrations in mice and that adiponectin reduces plasma triglyceride concentration in mice by increasing skeletal muscle lipoprotein lipase activity and VLDL receptor and consequently VLDL-triglyceride catabolism. Therefore, orally administered Oct may improve hypertriglyceridemia and the increase in serum FFA concentration in HFD-fed rats through the above-described improvement of hypoadiponectinemia.

Excessive fructose intake induces hepatic de novo lipogenesis contributing to hepatic triglyceride accumulation, i.e., nonalcoholic fatty liver disease, in association with insulin resistance in humans. ${ }^{(13,39)}$ In addition, it is known that cholesterol synthesis associated with hepatic lipid content is dependent on fructose intake rather than glucose intake in healthy humans. ${ }^{(40)}$ There are several reports showing increased hepatic triglyceride and/or cholesterol contents in rats fed a diet containing 60 or $65 \%$ fructose for 3 or 4 weeks. . $^{(22,23,25,32,33)}$ In the present study, rats fed HFD for 3 and 4 weeks had significant increases in hepatic triglyceride and cholesterol contents when compared with the corresponding CD-fed rats, although the increases in hepatic triglyceride and cholesterol contents tended to be larger at 4-week HFD feeding than at 3-week HFD feeding. Daily oral administration of Oct (10 or $50 \mathrm{mg} / \mathrm{kg}$ body weight) for the last oneweek HFD feeding prevented the increase in hepatic triglyceride content at 4-week HFD feeding completely. In addition, this prevention by administered Oct was more effectively at its higher dose than at its lower dose. The administration of Oct at both doses prevented the increase in hepatic cholesterol content at 4-week HFD feeding significantly. It is known that hyperuricemia induced by excessive fructose intake is due to the secretion of uric acid from liver tissues where excessive uric acid is produced through the degradation of AMP which is formed through the metabolism of absorbed fructose to fructose 1-phosphate through a rapid phosphorylation with ATP via fructokinase (ketohexokinase). ${ }^{(41)}$ It is also known that uric acid accelerates fructose metabolism by stimulating fructokinase in hepatocytes, resulting in the development of fatty liver. ${ }^{(42)}$ It has been reported that uric acid induces hepatic triglyceride accumulation by generating mitochondrial oxidative stress and by activating sterol regulatory element binding protein-1c via endoplasmic reticulum stress. ${ }^{(43,44)}$ Therefore, it is conceivable that orally administered Oct improves hepatic triglyceride accumulation in HFD-fed rats through the above-described improvement of hyperuricemia. We have demonstrated that a single oral administration of Oct $(50 \mathrm{mg} / \mathrm{kg}$ body weight) to normal rats without hyperuricemia reduces both serum triglyceride concentration and hepatic triglyceride content. ${ }^{(6)}$ Therefore, there is a possibility that orally administered Oct improves hepatic triglyceride accumulation in HFD-fed rats not only through the improvement of hyperuricemia but also through other mechanisms such as the regulation of triglyceride synthesis. Singh and Porter ${ }^{(45)}$ have reported that Oct cannot reduce the activity of 3-hydroxy-3-methylglutaryl coenzyme A (HMG-CoA) reductase, a rate-limiting enzyme in cholesterol synthesis, in rat hepatoma cells. On the other hand, Menéndez et al. ${ }^{(46)}$ have reported that DOO3, a mixture of very long chain saturated fatty acids, which contains octacosanoic acid in the most abundant, exerts a suppressive effect on cholesterol synthesis in cultured fibroblasts under metabolic conditions in which the upregulation of $\mathrm{HMG}-\mathrm{CoA}$ reductase is enhanced. Moreover, Menéndez et al. ${ }^{(47)}$ have shown that Oct present in orally administered polycosanol is metabolized to octacoanoic acid in the liver of rats. Therefore, there seems to be a possibility that orally administered Oct improves cholesterol accumulation in the liver of HFD-fed rats by inhibiting enhanced up-regulation of HMG-CoA reductase via octacosanic acid produced as the metabolite of Oct in the tissue. Thus, orally administered Oct was found to improve hepatic lipid accumulation associated with the feature of MetS in HFD-fed rats. From these findings, it is suggested that orally administered Oct could cause the above-described improvement of hepatic hypertrophy in HFD-fed rats by reducing lipid accumulation in the liver. It has been demonstrated that high fructose intake causes not only activation of hepatic de novo lipogenesis in association with hepatic insulin resistance but also increases in hepatic VLDL production and secretion, which lead to hypertriglyceridemia. ${ }^{(13)}$ Not only triglyceride but also cholesterol is incorporated into VLDL produced in the liver. VLDL secreted from the liver to the blood stream is converted to low-density lipoprotein carrying cholesterol via the formation of intermediatedensity lipoprotein following the degradation of triglyceride present in VLDL by lipoprotein lipase. Therefore, these findings allow us to assume that orally administered Oct improves the above-described dyslipidemia including hypertriglyceridemia and hypercholesterolemia in HFD-fed rats through its improving effect on the accumulation of triglyceride and cholesterol in the liver. It has been shown that impaired endothelial nitric oxide synthase expression is associated with hepatic triglyceride accumulation in rats with high fructose corn syrup intake. ${ }^{(48)}$ It has also been shown that lower nitric oxide level leads to hypertriglyceridemia by lowering hepatic fatty acid oxidation and by increasing serum triglyceride level in rats fed diet containing a nitric oxide synthase inhibitor. ${ }^{(49)}$ A recent report ${ }^{(50)}$ has shown that short-term supplementation with nitrate, an oxidation product of nitric oxide, decreases hepatic triglyceride content in genetic and dietaryinduced mouse models of obesity. ${ }^{(50)}$ Therefore, a further study is needed to verify whether orally administered Oct improves triglyceride accumulation in the liver of HFD-fed rats by affecting nitric oxide synthesis and nitric oxide and nitrate levels in the tissue.

Excessive fructose consumption is known to induce hypertension as the feature of MetS in humans. ${ }^{(15)}$ It has been reported that rats fed a diet containing 60 or $65 \%$ fructose for 3 or 4 weeks have elevated SBP, ${ }^{(17,18,25,32)}$ although our previous report ${ }^{(22)}$ has shown no change in SBP in rats fed a diet containing $60 \%$ fructose for 4 weeks. In the present study, rats fed HFD for 3 and 4 weeks had no elevation of SBP. Daily administration of Oct (10 or $50 \mathrm{mg} / \mathrm{kg}$ body weight) to HFD-fed rats for the last one-week HFD feeding had no effect on SBP at 4-week HFD feeding. However, it is uncertain at present whether orally administered Oct exerts an antihypertensive action in rats with high fructoseinduced hypertension. Therefore, further investigation is needed to clarify the effect of Oct on high fructose-induced hypertension.

Hokayem et al. ${ }^{(14)}$ have demonstrated that 6-day fructose ingestion in a drinking water $(3 \mathrm{~g} / \mathrm{kg}$ body weight per day) induces 
oxidative stress evaluated using urinary F2-isoprostane excretion, muscle LPO level, and muscle protein carboxylation in healthy overweight/obese first-degree relatives of type 2 diabetic patients. Yokozawa et al. ${ }^{(25)}$ have reported increases in serum and hepatic LPO levels in rats fed a diet containing $65 \%$ fructose for 3 weeks. Our previous report ${ }^{(22)}$ has shown that feeding of a diet containing $60 \%$ fructose to rats for 4 weeks causes an increase in serum LPO level without altering hepatic LPO and GSH levels. In contrast, Srividhya and Anuradha ${ }^{(51)}$ have reported that an increase in LPO level and decreases in GSH and vitamins $\mathrm{C}$ and $\mathrm{E}$ levels occur in the liver of rats fed a diet containing $60 \%$ fructose for 4 weeks. In the present study, 3- or 4-week HFD feeding to rats caused a significant increase in serum LPO concentration. The increase in serum LPO concentration was larger at 4-week HFD feeding than at 3-week HFD feeding. Thus, systemic oxidative stress occurred as the feature of MetS in HFD-fed rats. In HFD-fed rats with daily oral administration of Oct (10 or $50 \mathrm{mg} / \mathrm{kg}$ body weight) for the last one week of HFD feeding, the increased serum LPO concentration was attenuated significantly. In addition, there was no significant difference in serum LPO concentration between HFD rats with the higher dose of Oct and the corresponding CDfed rats. Thus, systemic oxidative stress associated with the feature of MetS in HFD-fed rats was found to be improved by orally administered Oct. Feeding of HFD to rats for 4 weeks caused a significant decrease in hepatic vitamin $\mathrm{C}$ content without affecting hepatic GSH content. Hepatic LPO content in rats fed HFD for 4 weeks tended to be higher than that in the corresponding CD-fed rats. The decreased hepatic vitamin C content in rats fed HFD for 4 weeks was returned to the level of the corresponding CD-fed rats by oral administration of Oct at a dose of 10 or $50 \mathrm{mg} / \mathrm{kg}$ body weight for the last one-week HFD feeding. The higher dose of Oct reduced hepatic LPO content in HFD-fed rats below the level of the corresponding CD-fed rats. The lower and higher doses of Oct elevated hepatic GSH content in HFD-fed rats above the level of the corresponding CD-fed rats. These findings allow us to think that orally administered Oct can improve hepatic oxidative stress associated with the feature of MetS in HFD-fed rats. We have demonstrated that Oct has no inhibitory effect on lipid peroxidation induced by a free radical generator, 2,2'-azobis(2-amidinopropane), in rat liver microsomes in vitro. ${ }^{(7)}$ It is known that GSH exerts antioxidant action by scavenging reactive oxygen species such as superoxide radical and hydroxyl radical directly, by working as the co-substrate for glutathione peroxidase to metabolize hydrogen peroxide and lipid hydroperoxides, and by regenerating vitamin $\mathrm{C}$ and $\mathrm{E}$ from their oxidized form. ${ }^{(52)}$ We have demonstrated that normal rats with a single oral administration of Oct $(10,50$ or $100 \mathrm{mg} / \mathrm{kg}$ body weight) have a dose-dependent increase in GSH content with a dose-dependent decrease in LPO content in the liver. ${ }^{(7)}$ From these findings, it is conceivable that orally administered Oct can

\section{References}

1 Taylor JC, Papport L, Lockwod GB. Octacosanol in human health. Nutrition 2003; 19: 192-195.

2 Kato S, Karino K, Hasegawa S, et al. Octacosanol affects lipid metabolism in rats fed on a high-fat diet. Br J Nutr 1995; 73: 433-441.

3 Xu Z, Fitz E, Riediger N, Moghadasian MH. Dietary octacosanol reduces plasma triacylycerol levels but not atherogenesis in apolipoprotein Eknockout mice. Nutr Res 2007; 27: 212-217.

4 Ohashi K, Ishikawa H, Ohta Y. Octacosanol ameliorates hyperlipidemia and oxidative stress in KKAy mice with type 2 diabetes. J Anal Bio-Sci 2011; 34: 223-233

5 Sharma R, Matsuzaki T, Kaushik M, et al. Octacosanol and policosanol prevent high-fat diet-induced obesity and metabolic disorders by activating brown adipose tissue and improving liver metabolism. Sci Rep 2019; 9: 5169.

6 Ohta Y, Sasaki E, Ishiguro I. Effect of oral octacosanol administration on hepatic triglyceride accumulation in rats with carbon tetrachloride-induced liver

improve oxidative stress associated with the feature of MetS in the liver of HFD-fed rats by elevating GSH content in the tissue. Therefore, further studies are needed to elucidate the mechanism by which orally administered Oct elevates hepatic GSH content in HFD-fed rats, resulting in the reduction of oxidative stress in the tissue.

In conclusion, the results obtained from the present study indicate that orally administered Oct improves the features of MetS such as hypoadiponectinemia, hyperuricemia, dyslipidemia, and oxidative stress without affecting hyperinsulinemia and insulin resistance in HFD-fed rats. Therefore, it seems to be postulated that supplemental Oct administration is useful to improve some features of MetS for humans with MetS. However, further studies using several animal models of MetS are needed to elucidate the mechanism by which orally administered Oct improves various features of MetS.

\section{Author Contributions}

Author contributions were as follows: study design ( $\mathrm{KO}$ and $\mathrm{YO})$, data collection (KO, HI, and $\mathrm{AK})$, statistical analysis (KO, $\mathrm{HI}$, and $\mathrm{AK}$ ), data interpretation ( $\mathrm{KO}$ and $\mathrm{YO}$ ), manuscript preparation ( $\mathrm{KO}$ and $\mathrm{YO}$ ), and fund collection $(\mathrm{KO})$.

\section{Acknowledgments}

This work was supposed by a grant from the Research Foundation of Fujita Health University.

\section{Abbreviations}

CD

DTNB

EDTA

FFA

GSH

HDL

HFD

HOMA-IR

MDA

MetS

LPO

Oct

SBP

TBA

VLDL

\section{Conflict of Interest}

No potential conflicts of interest were disclosed.

control diet 5,5'-dithiobis(2-nitrobenzoic acid) ethylenediaminetetraacetic acid free fatty acids reduced glutathione high-density lipoprotein high fructose diet homeostasis model assessment for insulin resistance malondialdehyde metabolic syndrome lipid peroxide octacosanol systolic blood pressure 2-thiobarbituric acid very low-density lipoprotein injury. Med Biol (Igaku to Seibutsugaku) 1997; 134: 185-189. (in Japanese) 7 Ohta Y, Ohashi K, Matsura T, Tokunaga K, Kitagawa A, Yamada K. Octacosanol attenuates disrupted hepatic reactive oxygen species metabolism associated with acute liver injury progression in rats intoxicated with carbon tetrachloride. J Clin Biochem Nutr 2008; 42: 118-125.

8 Eckel RH, Grundy SM, Zimmet PZ. The metabolic syndrome. Lancet 2005; 365: 1415-1428.

9 Zafar U, Khaliq S, Ahmad HU, Manzoor S, Lone KP. Metabolic syndrome: an update criteria, pathogenesis, and genetic links. Hormones 2018; 17: 299 313.

10 Roberts CK, Sindhu KK. Oxidative stress and metabolic syndrome. Life Sci 2009; 84: 705-712.

11 Francisqueti FV, Chaverini LC, Santos KC, et al. The role of oxidative stress on the pathophysiology of metabolic syndrome. Rev Assoc Med Bras (1992) 2017; 63: 85-91. 
12 Miller A, Adeli K. Dietary fructose and the metabolic syndrome. Curr Opin Gastroenterol 2008; 24: 204-209.

13 Stanhope KL, Havel PJ. Fructose consumption: potential mechanisms for its effects to increase visceral adiposity and induce dyslipidemia and insulin resistance. Curr Opin Lipidol 2006; 19: 16-24.

14 Hokayem M, Blond E, Vidal H, et al. Grape polyphenols prevent fructoseinduced oxidative stress and insulin resistance in first-degree relatives of type 2 diabetic patients. Diabetes Care 2013; 36: 1454-1461.

15 Kelishadi R, Mansourian M, Heidari-Beni M. Association of fructose consumption and components of metabolic syndrome in human studies: a systematic review and meta-analysis. Nutrition 2014; 30: 503-510.

16 ter Horst KW, Schene MR, Holman R, Romijn JA, Serlie MJ. Effect of fructose consumption on insulin sensitivity in nondiabetic subjects: a systematic review and meta-analysis of diet-intervention. Am J Clin Nutr 2016; 104: 1562-1576.

17 Faure P, Rossini E, Lafond JL, Richard MJ, Favier A, Halimi S. Vitamin E improves the free radical defense system potential and insulin sensitivity of rats fed high fructose diets. J Nutr 1997; 127: 103-107.

18 Delbosc S, Paizanis E, Magous R, et al. Involvement of oxidative stress and NADPH oxidase activation in the development of cardiovascular complications in a model of insulin resistance, the fructose-fed rat. Atherosclerosis 2005; 179: 43-49.

19 Tran LT, Yuen VG, McNeill JH. The fructose-fed rat: a review on the mechanisms of fructose-induced insulin resistance and hypertension. Mol Cell Biochem 2009; 332: 145-159.

20 Polakof S, Dardevet D, Lyan B, et al. Time course of molecular and metabolic events in the development of insulin resistance in fructose-fed rats. $J$ Proteome Res 2016; 15: 1862-1874.

21 Korandji C, Zeller M, Guilland JC, et al. Time course of asymmetric dimethylarginine (ADMA) and oxidative stress in fructose-hypertensive rats: a model related to metabolic syndrome. Atherosclerosis $2011 ; 214$ : 310-315.

22 Kitagawa A, Ohta Y, Ohashi K. Melatonin improves metabolic syndrome induced by high fructose intake in rats. J Pineal Res 2012; 52: 403-413.

23 Busserolles J, Gueux E, Rock E, Dermigné C, Mazur A, Rayssiguier Y. Oligofructose protects against the hypertriglyceridemia and pro-oxidative effects of a high fructose diet in rats. J Nutr 2003; 133: 1903-1908.

24 Joyeux-Faure M, Rossini E, Ribuot C, Faure P. Fructose-fed rat hearts are protected against ischemia-reperfusion injury. Exp Biol Med (Maywood) 2006; 231: 456-462.

25 Yokozawa T, Kim HJ, Yamabe N, Okamoto T, Cho EJ. The protective role of Kangen-karyu against fructose-induced metabolic syndrome in a rat model. J Pharm Pharmacol 2007; 59: 1271-1278.

26 Matthews DR, Hosker JP, Rudenski AS, Naylor BA, Treacher DF, Turner RC. Homeostasis model assessment: insulin and beta-cell function from fasting plasma glucose and insulin concentration in man. Diabetologia 1985; 28: 412-419.

27 Yagi K. A simple fluorometric assay for lipoperoxide in blood plasma. Biochem Med 1976; 15: 212-216.

28 Folch J, Lees M, Sloane Stanley GH. A simple method for the isolation and purification of total lipids from animal tissues. J Biol Chem 1957; 226: $497-$ 509.

29 Ohkawa H, Ohishi N, Yagi K. Assay for lipid peroxide in animal tissues by thiobarbituric acid reaction. Anal Biochem 1979; 95: 351-358.

30 Sedlak J, Lindsay RH. Estimation of total, protein-bound, and non-protein sulfhydryl groups in animal tissues with Ellman's reagent. Anal Biochem 1968; 25: 192-205.

31 Zannoni V, Lynch M, Goldstein S, Sato P. A rapid micromethod for the determination of ascorbic acid in plasma and tissues. Biochem Med 1974; 11 : $41-48$.

32 Jaroslawska J, Juskierwicz J, Wroblewska M, Jurgonski A, Krol B, Zdunczyk Z. Polyphenol-rich strawberry pomace reduces serum and liver lipids and alters gastrointestinal metabolite formation in fructose-fed rats. J Nutr 2011; 141: $1777-1783$.

33 Kim HY, Okubo T, Juneja LR, Yokozawa T. The protective role of amla
(Emblica officinalis Gaertn.) against fructose-induced metabolic syndrome in a rat model. Br J Nutr 2010; 103: 502-512.

34 Perez-Pozo SE, Schold J, Nakagawa T, Sánchez-Lozada LG, Johnson RJ, Lillo JL. Excessive fructose intake induces the features of metabolic syndrome in healthy adult men: role of uric acid in the hypertensive response. Int J Obes (Lond) 2010; 34: 454-461.

35 Okamoto Y, Kihara S, Funahashi T, Matsuzawa Y, Libby P. Adiponectin: a key adipocytokine in metabolic syndrome. Clin Sci (Lond) 2006; 110: 267278.

36 Baldwin W, McRae S, Marek G, et al. Hyperuricemia as a mediator of the proinflammatory endocrine imbalance in the adipose tissue in a murine model of the metabolic syndrome. Diabetes $2011 ; \mathbf{6 0}$ : 1258-1269.

37 Lê KA, Faeh D, Stettler R, et al. A 4-wk high-fructose diet alters lipid metabolism without affecting insulin sensitivity or ectopic lipids in healthy humans. Am J Clin Nutr 2006; 84: 1374-1379.

38 Qiao L, Zou C, van der Wesathuyzen DR, Shao J. Adiponectin reduced plasma triglyceride by increasing VLDL triglyceride catabolism. Diabetes 2008; 57: 1824-1833.

39 Softic S, Cohen DE, Kahn CR. Role of dietary fructose and hepatic de novo lipogenesis in fatty liver disease. Dig Dis Sci 2016; 61: 1282-1293.

40 Silbernagel G, Lütjohann D, Machann J, et al. Cholesterol synthesis is associated with hepatic lipid content and dependent on fructose/glucose intake in healthy humans. Exp Diabetes Res 2012; 2012: 361863.

41 Hallfrisch J. Metabolic effect of dietary fructose. FASEB J 1990; 4: 2652 2660.

42 Lanaspa MA, Sanchez-Lozada LG, Cicerchi C, et al. Uric acid stimulates fructokinase and accelerates fructose metabolism in the development of fatty liver. PLoS One 2012; 7: e47948.

43 Lanaspa MA, Sanchez-Lozada LG, Choi YJ, et al. Uric acid induces hepatic steatosis by generation of mitochondrial oxidative stress: potential role in fructose-dependent and -independent fatty liver. J Biol Chem 2012; 287: 40732-40744.

44 Choi YJ, Shin HS, Choi HS, et al. Uric acid induces fat accumulation via generation of endoplasmic reticulum stress and SREBP-1c activation in hepatocytes. Lab Invest 2014; 94: 1114-1125.

45 Singh DK, Li L, Porter TD. Policosanol inhibits cholesterol synthesis in hepatoma cells by activation of AMP-kinase. J Pharmacol Exp Ther 2006; 318: $1020-1026$.

46 Menéndez R, Más R, Amor AM, Rodeiros I, Gonzalez RM, Alfonso JL. Inhibition of cholesterol biosynthesis in cultured fibroblasts by D003, a mixture of very long chain saturated fatty acids. Pharmacol Res 2001; 44: 299-304.

47 Menéndez R, Marrero D, Más R, Fernández I, González L, González RM. In vivo and in vitro study of octacosanol metabolism. Arch Med Res 2005; 36: $113-119$.

48 Said G, Ergin V, Yilmaz G, et al. High-fructose corn syrup-induced hepatic dysfunction in rats: improving effect of resveratrol. Eur J Nutr 2015; 54: 895904.

49 Khedara A, Kawai Y, Kayashita J, Kato N. Feeding rats the nitric oxide synthase inhibitor, L-N ${ }^{\omega}$ nitroarginine, elevates serum triglyceride and cholesterol and lowers hepatic fatty acid oxidation. J Nutr 1996; 126: $2563-$ 2567.

50 Fischer A, Lüersen K, Schultheiß G, et al. Supplementation with nitrate only modestly affect lipid and glucose metabolism in genetic and dietary-induced murine models of obesity. J Clin Biochem Nutr 2020; 66: 24-35.

51 Srividhya S, Anuradha CV. Metformin improves liver antioxidant potential in rats fed a high-fructose diet. Asia Pac J Clin Nutr 2002; 11: 319-322.

52 Gul M, Kutay FZ, Temocin S, Hanninen O. Cellular and clinical implication of glutathione. Indian J Exp Biol 2000; 38: 625-634.

This is an open access article distributed under the terms of the Creative Commons Attribution-NonCommercial-NoDerivatives License (http://creativecommons.org/licenses/by-nc-nd/4.0/). 